\title{
orrespondence
}

\section{Comparison between the Abbott RealTime High Risk HPV assay and the Hybrid Capture 2 assay for detecting high-risk human papillomavirus DNA in cervical specimens}

The new Abbott RealTime High Risk HPV assay is a qualitative real-time PCR assay that detects 14 human papillomavirus (HPV) genotypes and simultaneously identifies women infected with HPV-16 and -18. These two high-risk HPV genotypes are responsible for approximately $70 \%$ of cervical cancers worldwide (Bosch et al., 2008; Muñoz et al., 2003, 2004; Seme et al., 2006).

The aim of this study was to compare the analytical and clinical performance of the Abbott PCR assay with the USA Food and Drug Administration-approved screening assay Hybrid Capture 2 (HC2) (Qiagen) in detecting DNA from high-risk HPV genotypes in clinical samples collected in the context of the routine laboratory setting.

A total of 108 women were enrolled in the study whose cervical specimens were tested at the Educational Public Health Institute of Split and Dalmatia County. The presence of HPV DNA in the clinical samples was assessed based on the concordance between both assays. Discordant samples were further genotyped by using the INNO-LiPA HPV Genotyping Extra test (Innogenetics) at the University Hospital for Infectious Diseases in Zagreb.

A total of 48 of 108 (44.4\%) samples tested positive for high-risk genotypes in the Abbott PCR assay, whereas 45 of 108 (41.7\%) samples tested positive with HC2 assay. Forty-two samples tested positive by both assays and fifty-seven samples were negative by both assays. A total of 6 of the 42 samples that tested positive with both assays had normal cytology, 18 samples showed cervical intraepithelial neoplasia levis (CIN1) and 15 samples showed cervical intraepithelial neoplasia media and gravis $\geqslant$ CIN2 (data not available for 3 samples). The majority of samples that tested negative by both assays had normal cytology (30/57) or CIN1 (21/57) (data not available for 6 samples). Two of the nine discordant samples that tested positive by Abbott PCR and negative by $\mathrm{HC} 2$ assay showed CIN2.

The results of the nine discordant samples were resolved by INNO-LiPA test (Table 1). HPV was detected in two of three discordant samples that tested positive with the HC2 assay and negative with the Abbott PCR assay. These discordant samples were interpreted as HPV-positive and the result of Abbott PCR was interpreted as false negative.

Five of six samples that tested negative with HC2 and positive with Abbott PCR were shown to contain HPV DNA by INNO-LiPA test. These results were interpreted as false-negative results for HC2. The results by Abbott PCR and INNO-LiPA genotyping tests were completely concordant in two samples only (sample 4 and sample 8 ).

The presence of HPV in two of the nine discordant samples could not be confirmed by INNO-LiPA assay. One of those samples tested positive by $\mathrm{HC} 2$ only (and showed CIN1) and the other sample tested positive by Abbott PCR only (cytology data not available). These results were interpreted as false-positive results for HC2 and Abbott PCR, respectively. The percentages of HPV DNA-positive samples obtained in this study were high compared to an earlier study conducted in the same county [200 positive samples out of 570 samples with the HC2 assay (35\%)] (Kaliterna et al., 2007).

In the present study, based on the results of the Abbot PCR assay, HC2 assay and INNO-LiPA assay, 49 samples were considered HPV DNA positive. Therefore, the estimated analytical sensitivity of the Abbott PCR assay for detection of highrisk HPV infections was $96 \%$ (47 of 49 positive samples detected) and the analytical sensitivity of the HC2 assay was $90 \%$ (44 of 49 positive samples detected). The analytical specificity of both assays was $98 \%$ (both assays detected 58 of 59 negative samples). Clinical evaluation of newly developed candidate HPV DNA tests for cervical screening should focus on the ability to detect high-risk HPV infections that are associated or will develop into $\geqslant \mathrm{CIN} 2$, and to differentiate them from clinically insignificant transient infections as recommended by Meijer et al. (2009).

The presence of disease defined as a histology result of CIN2 or greater was detected in 17 of 108 patients included in this study (prevalence of disease $15.7 \%$ ). The clinical sensitivity of the Abbott PCR assay for detection of disease (patients with $\geqslant \mathrm{CIN} 2$ ) was $100 \%$, whereas the HC2 assay detected 15 of 17 patients with $\geqslant$ CIN2 (clinical sensitivity of $88.2 \%$ ).

A total of 80 samples tested in this study had normal cytology or CIN1 and were defined as disease negative. The Abbott PCR assay tested negative for 60 of 80 disease-negative samples (clinical specificity $75 \%$ ), whereas the HC2 assay tested negative for 63 of 80 diseasenegative samples (clinical specificity $79 \%$ ).

In conclusion, the Abbott PCR test is suitable for qualitative detection of HPV DNA from high-risk genotypes in clinical specimens and simultaneous identification of women infected with HPV-16/HPV-18. This assay showed better clinical sensitivity for detection of high-risk HPV infection and disease than the standard $\mathrm{HC} 2$ assay. However, the clinical significance of the additional positive HPV DNA results obtained by the Abbott PCR test needs to be confirmed in a long-term clinical follow up of the patients. Importantly, the failure of the Abbott PCR assay to detect HPV-18 in two clinical samples and HPV-51 in one clinical sample (in comparison with the INNO-LiPA test) needs to be further 
Table 1. HPV genotyping of HC2 and Abbott RealTime HR HPV assay discordant samples with the INNO-LiPA HPV Genotyping Extra test

\begin{tabular}{|lccl|}
\hline Sample & HC2 assay $^{*}$ & Abbott RealTime HR HPV assay $\dagger$ & INNO-LiPA HPV Genotyping Extra test $¥$ \\
\hline 1 & + & - & HPV positives \\
2 & + & - & HPV-52 \\
3 & + & - & HPV negative \\
4 & - & Other HR HPV & HPV-51 and HPV-54 \\
5 & - & Other HR HPV & HPV-18 and HPV-51 \\
6 & - & HPV-18 & HPV-18, HPV-73 and HPV-51 \\
7 & - & Other HR HPV & HPV-18, HPV-35 and HPV-51 \\
8 & - & HPV-18 & HPV-18 \\
9 & - & Other HR HPV & HPV negative \\
\hline
\end{tabular}

${ }^{\star}$ Positive results were the 13 highest risk HPV genotypes.

$\dagger$ Positive results were reported as HPV-16, HPV-18, other HR HPV (12 genotypes) or a combination of the three (mixed infection). $\ddagger$ The presence of HPV DNA (27 genotypes) was confirmed by gel electrophoresis and positive amplification control in the INNO-LiPA assay. \$Positive amplification control and gel electrophoresis but the genotype could not be determined.

evaluated by testing large numbers of clinical samples.

\section{Acknowledgements}

This study was supported in part by grants from the Croatian Ministry of Science, Education and Sports to Dr Zidovec Lepej and Dr Vince (grant numbers 143-10801160097 and 143-0000000-0117).

\section{Vanja Kaliterna, ${ }^{1}$ Snjezana Židovec Lepej $^{2}$ and Adriana Vince ${ }^{2}$}

${ }^{1}$ Educational Public Health Institute of Split and Dalmatia County, Vukovarska 46, 21000 Split, Croatia

${ }^{2}$ University Hospital for Infectious Diseases 'Dr Fran Mihaljevic', Zagreb, Croatia
Correspondence:

Snjezana Židovec Lepej

(snjezana.zidovec.lepej@bfm.hr)

Bosch, F. X., Burchell, A. N., Schiffman, M., Giuliano, A. R., De Sanjose, S., Bruni, L., Tortolero-Luna, G., Kjaer, S. K. \& Muñoz, N. (2008). Epidemiology and natural history of human papillomavirus infections and typespecific implications in cervical neoplasia. Vaccine 26, K1-K16.

Kaliterna, V., Anđelinović, Š., Pejković, L. \& Drmić, H. I. (2007). Human papillomavirus DNA typing in the cervical specimens among women of Split and Dalmatia County. Coll Antropol 31, 79-82.

Meijer, C. J. L. M., Berkhof, J., Castle, P. E., Hesselink, A. T., Franco, E. L., Ronco, G., Arbyn, M., Bosch, F. X., Cuzick, J. \& other authors (2009). Guidelines for human papillomavirus DNA test requirements for primary cervical cancer screening in women 30 years and older. Int J Cancer 124, 516-520.

Muñoz, N., Bosch, F. X., De Sanjosé, S., Herrero, R., Castellsagué, X., Shah, K. V., Snijders, P. J. F. \& Meijer, C. J. L. M., for the International Agency for Research on Cancer Multicenter Cervical Cancer Study Group (2003). Epidemiologic classification of human papillomavirus types associated with cervical cancer. N Engl J Med 348, 518-527.

Muñoz, N., Bosch, F. X., Castellsagué, X., Diaz, M., De Sanjose, S., Hammouda, D., Shah, K. V. \& Meijer, C. J. L. M. (2004). Against which human papillomavirus types shall we vaccinate and screen? The international perspective. Int $J$ Cancer 111, 278-285.

Seme, K., Fuis, K., Kocian, B. J. \& Poljak, M. (2006). Resolving repeatedly borderline results of Hybrid Capture 2 HPV DNA Test using polymerase chain reaction and genotyping. $J$ Virol Methods 134, 252-256. 